\title{
Postoperative radiation therapy following the incomplete resection of a non-small cell lung cancer
}

\author{
Jaehyeon Park, MD', Si Yeol Song, MD, PhD', Su Ssan Kim, MD, PhD', Sang-We Kim, MD, PhD², \\ Woo Sung Kim, MD, PhD², Seung-II Park, MD, PhD³, Dong Kwan Kim, MD, PhD³, \\ Yong-Hee Kim, MD, PhD'3 , Jongmoo Park, MD', Sang-wook Lee, MD, PhD', \\ Jong Hoon Kim, MD, PhD', Seung Do Ahn, MD, PhD', Eun Kyung Choi, MD, PhD \\ Departments of ${ }^{1}$ Radiation Oncology, ${ }^{2}$ Internal Medicine, and ${ }^{3}$ Thoracic and Cardiovascular Surgery, Asan Medical Center, \\ University of Ulsan College of Medicine, Seoul, Korea
}

Purpose: To review the results of postoperative radiation therapy (PORT) for residual non-small cell lung cancer (NSCLC) following surgical resection and evaluate multiple clinicopathologic prognostic factors.

Materials and Methods: A total of 58 patients, who completed scheduled PORT for positive resection margin, among 658 patients treated with PORT from January 2001 to November 2011 were retrospectively analyzed. Radiation therapy was started at 4 to 6 weeks after surgery. Chemotherapy was also administered to 35 patients, either sequentially or concurrently with PORT.

Results: The median age of patients was 63 years (range, 40 to 82 years). The postoperative pathological stage I NSCLC was diagnosed in $10(17.2 \%)$, stage II in $18(31.0 \%)$, and stage III in 30 patients (51.7\%). Squamous cell carcinoma was identified in 43 , adenocarcinoma in 10, large cell in 1, others in 4 patients. Microscopic residual disease (R1) was diagnosed in 55 patients (94.8\%), and the remaining three patients were diagnosed with gross residual disease (R2). The median dose of PORT was 59.4 Gy (range, 50.0 to $64.8 \mathrm{~Gy}$ ). Chemotherapy was administered to 35 patients (60\%), and the median follow-up time was 22.0 months (range, 6.0 to 84.0 months). The 3 -year locoregional relapse-free survival and distant metastasis-free survival rates were $82.1 \%$ and $52.9 \%$, respectively. The median overall survival was 23.8 months (range, 6.0 to 84.1 months), and the 3 -year overall survival rate was $58.2 \%$. Chemotherapy did not influence the failure pattern or survival outcome.

Conclusion: PORT is an effective modality for improving local tumor control in incompletely resected NSCLC patients. Major failure pattern was distant metastasis despite chemotherapy.

Keywords: Non-small-cell lung carcinoma, R1 resection, Microscopic residual disease, Postoperative radiotherapy

\section{Introduction}

Complete surgical resection is the treatment of choice for curing locally advanced non-small cell lung cancer (NSCLC). However, $1 \%$ to $17 \%$ of surgical resections for NSCLC are reportedly incomplete $[1,2]$. Incomplete resection is associated with increased local recurrence and negatively impacts on survival [2-5]. Postoperative radiation therapy (PORT) is essential for improving local tumor control in patients with incompletely resected NSCLC, and the National Comprehensive

Received 5 March 2014, Revised 11 April 2014, Accepted 9 June 2014.

Correspondence: Si Yeol Song, MD, PhD, Department of Radiation Oncology, Asan Medical Center, University of Ulsan College of Medicine, 88 Olympic-ro 43-gil, Songpa-gu, Seoul 138-736, Korea. Tel: +82-2-3010-4431, Fax: +82-2-3010-4427, E-mail: coocoori@gmail.com

(c) This is an Open Access article distributed under the terms of the Creative Commons Attribution Non-Commercial License (http://creativecommons.org/ licenses/by-nc/3.0/) which permits unrestricted non-commercial use, distribution, and reproduction in any medium, provided the original work is properly cited.

www.e-roj.org 
Cancer Network guidelines recommend combination chemoradiation therapy for incompletely resected, locally advanced NSCLC. The type of combination adjuvant treatment-sequential or concurrent chemoradiationis determined by the extent of residual disease, age and performance of patient, and pathological stage. Concurrent chemoradiation therapy (CCRT) may result in better local tumor control, but it can also result in additional treatmentrelated morbidity and poor tolerance in adjuvant settings following major surgery [6].

In this retrospective analysis, we reviewed the treatment outcomes of PORT for incompletely resected NSCLC and also evaluated multiple clinicopathologic prognostic factors.

\section{Materials and Methods}

Between January 2001 and November 2011, 658 patients with resected NSCLC received PORT at our institution. The medical records of these cases were fully reviewed, and 64 patients (9.7\%) had positive surgical margins noted in their pathological reports. Six patients were excluded because they received incomplete PORT during the early course of disease (progression of pneumonia during treatment in 5 , aggravation of acute renal failure which was unrelated with lung cancer treatment in 1). A total of 58 patients were then retrospectively reviewed, and this study was approved by the Institutional Review Board of our hospital.

All patients had pathologically confirmed NSCLC. R1 resection was defined as residual microscopic tumor on the surgical margins, and R2 resection was defined as residual macroscopic tumor determined by surgical findings or pathological reports. Pathological stage was determined according to the 7th edition of the American Joint Committee on Cancer. All patients underwent video-assisted thoracoscopic surgery-either thoracoscopic or open thoracotomy-with lobectomy, pneumonectomy, and sleeve resection. Mediastinal lymphadenectomy or systematic mediastinal lymph node sampling was also performed on all patients.

Computed tomography (CT)-simulation for PORT was done in all patients. The patients were placed in the supine position with arm-side, and immobilized using a pillow and vacuum mold. The target volume was delineated, which included the surgical stump, area of residual disease, ipsilateral hilum, and also covered both sides of the mediastinum, and the ipsilateral supraclavicular lymph nodes in patient with pN2. This area was defined as the clinical target volume (CTV). The planning target volume included 1-cm margins from the CTV. Boost radiation following initial treatment was only delivered to areas with residual disease after 50.4 Gy. Chemotherapy was administered according to nodal status. In most patients who received sequential chemoradiotherapy (CRT), radiation was delivered before or after systemic chemotherapy with monthly vinorebine + cisplatin. For CCRT, weekly docetaxel or paclitaxel + cisplatin was administered. Thirty-five of 58 patients (60.3\%) received chemotherapy.

Patients were evaluated every 3 months for 2 years after treatment, and every 6 months thereafter. Chest X-rays or chest CT was performed as part of the regular follow-up examinations. We used the National Cancer Institute Common Toxicity Criteria for Adverse Events (CTCAE) to assess side effects.

Overall survival (OS), locoregional relapse-free survival (LRFS), and distant metastasis-free survival (DMFS) were calculated using the Kaplan-Meier method. OS was defined from the day of surgical resection to the time of death or last follow-up examination. Local relapse was defined as any recurrence at the surgical resection margin, and regional relapse was defined as mediastinal recurrence (except at the surgical margins). Distant metastasis was defined as recurrence at any other organ or lung lobe. The log-rank method was used to compare sequential CRT with CCRT in terms of OS, LRFS, and DMFS. SPSS ver. 21.0 (IBM, Armonk, NY, USA) was used to perform the statistical analysis.

\section{Results}

Fifty-six patients (96.5\%) were male, and the median age was 63 years (range, 40 to 82 years). Squamous cell carcinoma was identified in 43 patients (74.1\%; the most common histological type), and 10 patients (17.2\%) were diagnosed with adenocarcinoma. Sixteen patients $(27.6 \%)$ received PORT due to positive resection margins without lymph node metastasis. Most patients were classified as stage II or IIIA (74.1\%). The patient characteristics are listed in Table 1 and the surgical characteristics are summarized in Table 2. Most incomplete resections were $\mathrm{R} 1$ ( 55 patients), and the most common site of residual disease was the bronchial resection margins (60.3\%).

All patients were treated with external beam radiation therapy. The median total dose administered to $\mathrm{R} 1$ resections was $59.4 \mathrm{~Gy}$ (range, 50.0 to $64.8 \mathrm{~Gy}$ ), and $63.0 \mathrm{~Gy}$ (range, 59.4 to $64.8 \mathrm{~Gy}$ ) was administered to $\mathrm{R} 2$ resections. Time between surgery and the start of radiation therapy ranged between 
Table 1. The characteristics of 58 incompletely resected NSCLC patients

\begin{tabular}{lc}
\hline \multicolumn{1}{c}{ Variable } & No. of patients $(\%)$ \\
\hline Age (yr), median (range) & $63(40-82)$ \\
Gender & \\
Male & $56(97.0)$ \\
Female & $2(3.0)$ \\
Histology & \\
Squamous cell carcinoma & $43(74.1)$ \\
Adenocarcinoma & $10(17.2)$ \\
Large cell & $1(1.7)$ \\
Others & $4(6.8)$ \\
T stage & \\
1 & $8(13.8)$ \\
2 & $30(51.7)$ \\
3 & $11(19.0)$ \\
4 & $9(15.5)$ \\
N stage & \\
0 & $16(27.6)$ \\
1 & $17(29.3)$ \\
2 & $25(43.1)$ \\
Stage & \\
I & $10(17.2)$ \\
II & $18(31.0)$ \\
IIIA & $25(43.1)$ \\
IIIB & $5(8.6)$ \\
\hline C n non-small cell &
\end{tabular}

NSCLC, non-small cell lung cancer.

a) According to the 7th edition of the American Joint Committee on Cancer.

0.9 to 5.2 months. Patients with adjuvant chemotherapy followed by RT had 4 months more interval between surgery and the start of RT (Table 3 ). Thirty-five patients received chemotherapy, including 3 patients who received chemotherapy before surgery. Twelve patients (20.6\%) were treated with adjuvant CCRT, and 20 patients (34.5\%) received sequential CRT (RT followed by chemotherapy or chemotherapy followed by RT) (Table 4).

The median follow-up period was 22.0 months (range, 6.0 to 84.0 months). The median overall survival was 23.8 months (range, 6.0 to 84.1 months), and the 3 -year OS rate was 49.3\% (Fig. 1). Eight patients (13.8\%) developed locoregional recurrence, and the resection margins were the most common site (7 of 8 patients). Twenty-three patients (39.7\%) were diagnosed with distant metastasis. The 3-year LRFS and DMFS rates were $82.1 \%$ and $52.9 \%$, respectively (Fig. 2). According to univariate analysis, stage, lymphovascular invasion, and pathological type were significant prognostic factors
Table 2. The characteristics of surgery

\begin{tabular}{cc}
\hline Variable & No. of patients (\%) \\
\hline Type of surgery & $33(56.9)$ \\
Lobectomy & $11(19.0)$ \\
Pneumonectomy & $10(17.2)$ \\
Bilobectomy & $4(6.9)$ \\
Sleeve lobectomy & \\
Extent of residual disease & $55(94.8)$ \\
R1 resection & $35(60.3)$ \\
Bronchial & $8(13.8)$ \\
Peribronchial & $4(6.9)$ \\
Chest wall & $10(17.2)$ \\
Mediastinum & $1(1.7)$ \\
Bronchial \&t peribronchial & $3(5.2)$ \\
R2 resection & \\
Other risk factors $(-/+)$ & $32(55.2) / 26(44.8)$ \\
LVI & $48(82.8) / 10(17.2)$ \\
PNI & $38(65.5) / 20(34.5)$ \\
ENE
\end{tabular}

LVI, lymphovascular invasion; PNI, perineural invasion; ENE, extranodal extension.

Table 3. The characteristics of radiation therapy (RT)

\begin{tabular}{cc}
\hline Variable & Value \\
\hline Total dose (Gy) & \\
R1 resection & $59.4(50.0-64.8)$ \\
Fraction size & $1.8-2.0$ \\
R2 resection & $63.0(59.4-64.8)$ \\
Fraction size & $1.8-2.0$ \\
Surgery-RT interval (mo) & $1.1(0.9-5.2)$ \\
RT duration (day) & $48(36-60)$ \\
\hline
\end{tabular}

Values are presented as median (range).

Table 4. The characteristics of chemotherapy

\begin{tabular}{cc}
\hline Variable & No. of patients $(\%)$ \\
\hline No chemotherapy & $23(39.7)$ \\
Chemotherapy & $35(60.3)$ \\
Adjuvant & \\
Concurrent & $12(20.6)$ \\
RT $\rightarrow$ CTx & $16(27.6)$ \\
CTx $\rightarrow$ RT & $4(6.9)$ \\
Neoadjuvant & $3(5.2)$
\end{tabular}

$\mathrm{RT}$, radiation therapy; CTx, chemotherapy.

associated with OS, LRFS, and DMFS, respectively (Table 5).

There were no differences between the CCRT and sequential CRT groups in terms of tumor characteristics (Table 6). Both 
the CCRT and sequential CRT groups demonstrated better local control compared with the RT-alone group (Table 7). The CCRT group demonstrated relatively superior local tumor control than sequential CRT group, but this finding was not statistically significant ( $p=0.23$ ) (Fig. 3). Distant metastasis was a major pattern of failure detected in both groups despite combination chemotherapy. The distant failure rate was 50\% in both the CCRT and sequential CRT groups. No patient developed severe acute or late toxicity greater than grade 3 . Twenty-seven patients (46.5\%) demonstrated acute grade 2 toxicity, and the most common symptom was esophagitis. Late grade 2 lung toxicity was only observed in 2 patients.

\section{Discussion and Conclusion}

Incomplete resection might be a negative prognostic factor for local tumor control and survival in patients with locally

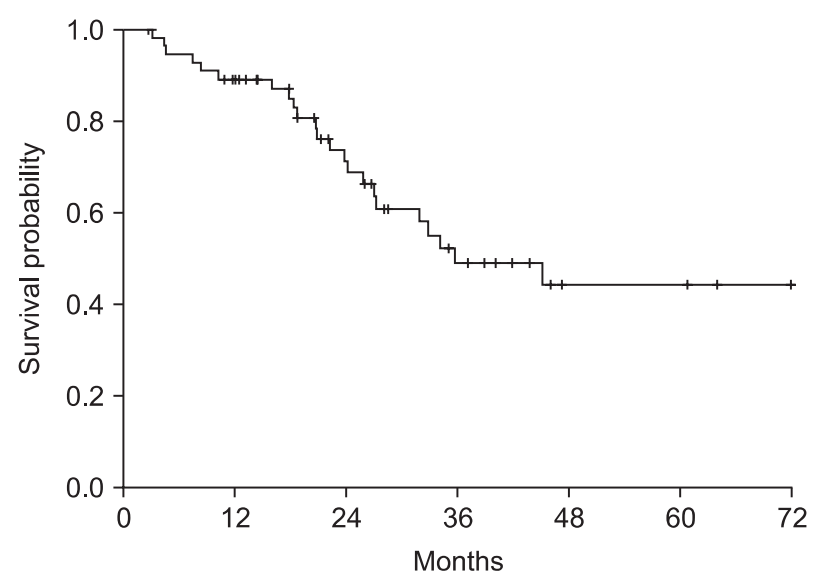

Fig. 1. Overall survival rate of all patients.

\section{A}

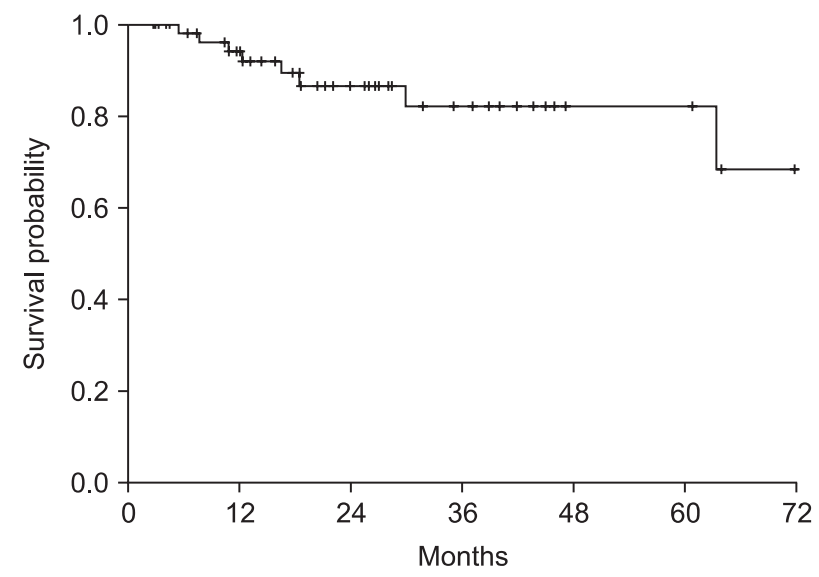

advanced NSCLC. Wind et al. [7] previously reported 1-year and 5 -year OS rates of $20 \%$ to $50 \%$ and $0 \%$ to $20 \%$, respectively, suggesting that residual disease in the peribronchial resection margins is associated with mediastinal lymph node metastasis. Riquet et al. [3] reported poor survival in patients with incomplete resection in comparison with complete resection (5-year OS: 20\% vs. 46\%, respectively; $p<0.001$ ).

Some studies report that administering PORT to patients with incomplete resection margins improves treatment outcomes. Ghiribelli et al. [8] reported 3-year OS and local control rates of $40 \%$ and $27.5 \%$, respectively, in incompletely resected NSCLC patients who received PORT without chemotherapy. Ohguri et al. [2] also reported that 5-year OS, local control, disease-free, and DMFS rates were 56\%,63\%, 37\%, and 49\%, respectively, in patients with incomplete resection margins who received PORT. The study of Ohguri et al. [2] reported a high local tumor control rate, although 44\% of their reviewed patients had R2 resection margins and the most common failure pattern was distant metastasis. These authors emphasized the necessity of effective systemic control.

In this retrospective study, PORT with chemotherapy resulted in high local tumor control (3-year LRFS, 82.9\%) in patients with incompletely resected NSCLC. This indicates better local tumor control than previously reported outcomes due to the high proportion of patients with $\mathrm{R} 1$ resection margins and combination chemoradiation. The target volume in all of our patients was defined for elective nodal irradiation (ENI), although the extent varied depending on the extent of the involved lymph nodes. This is probably why isolated regional relapse was observed in only one case in our study. OlszynaSerementa et al. [9] previously compared ENI + PORT and omitting ENI for the treatment of incompletely resected tumors. No statistical difference was found in the OS or local

B

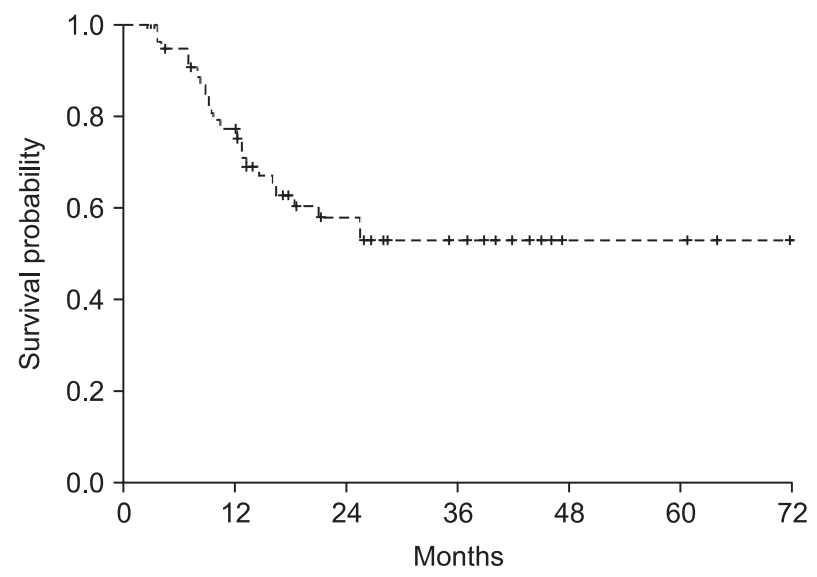

Fig. 2. (A) Locoregional relapse-free survival rate and (B) distant metastasis-free survival rate of all patients. 
Table 5. Univariate analysis of OS, LRFS, and DMFS

\begin{tabular}{|c|c|c|c|c|}
\hline \multirow{2}{*}{ Variable } & \multirow{2}{*}{ No. } & \multicolumn{3}{|c|}{ Univariate ( $p$-value) } \\
\hline & & OS & LRFS & DMFS \\
\hline Age (yr) & & 0.62 & 0.76 & 0.39 \\
\hline$<63$ & 28 & & & \\
\hline$\geq 63$ & 30 & & & \\
\hline Pathology & & 0.17 & 0.34 & $<0.01$ \\
\hline Non-adenocarcinoma & 48 & & & \\
\hline Adenocarcinoma & 10 & & & \\
\hline pT stage & & 0.23 & 0.94 & 0.56 \\
\hline $\mathrm{T} 1-2$ & 38 & & & \\
\hline T3-4 & 20 & & & \\
\hline pN stage & & 0.72 & 0.91 & 0.20 \\
\hline No-1 & 34 & & & \\
\hline N2-3 & 24 & & & \\
\hline Stage & & 0.04 & 0.25 & 0.29 \\
\hline I & 10 & & & \\
\hline$\|$ & 16 & & & \\
\hline III & 32 & & & \\
\hline ENE & & 0.78 & 0.63 & 0.55 \\
\hline Negative & 38 & & & \\
\hline Positive & 20 & & & \\
\hline LVI & & 0.63 & $<0.05$ & 0.44 \\
\hline Negative & 32 & & & \\
\hline Positive & 26 & & & \\
\hline PNI & & 0.26 & 0.76 & 0.42 \\
\hline Negative & 48 & & & \\
\hline Positive & 10 & & & \\
\hline RT dose (Gy) & & 0.27 & 0.12 & 0.27 \\
\hline$<60$ & 48 & & & \\
\hline$\geq 60$ & 10 & & & \\
\hline Chemotherapy & & 0.73 & 0.43 & 0.18 \\
\hline No & 23 & & & \\
\hline Yes & 35 & & & \\
\hline
\end{tabular}

OS, overall survival; LRFS, locoregional relapse-free survival; DMFS, distant metastasis-free survival; ENE, extranodal extension; LVI, lymphovascular invasion; PNI, perineural invasion; RT, radiation therapy.

control between the two groups in that report, but higher local recurrence (7.5\% vs $22.5 \%$, respectively) and isolated nodal failure $(7.5 \%$ vs $13.5 \%)$ were noted in patients who did not receive $\mathrm{ENI}$.

Most previous studies have reported distant metastasis from incompletely resected NSCLC as a major failure pattern in this setting. According to univariate analysis of our study, adenocarcinoma is the only significant risk factor for DMFS. Ohguri et al. [2] reported that adenocarcinoma is a significant predictor of distant metastasis in patients with incompletely resected NSCLC who received PORT ( $p=0.03$ ). These data suggested that effective systemic control is essential for improving survival because local recurrence and residual disease can be controlled using adjuvant radiation therapy. According to our current study results, combination adjuvant chemoradiotherapy demonstrates good local tumor control of incompletely resected NSCLC, but distant metastasis is still a major failure pattern. The results were similar regardless of the type of combination chemoradiation used (i.e., sequential CRT or CCRT).

There have been no earlier studies comparing postoperative concurrent and sequential CRT. The differences between postoperative concurrent and sequential CRT could be inferred from the Cochrane database, although this is the meta-analysis of definitive setting. According to the Cochrane database which compares definitive CCRT and 
Table 6. Tumor characteristics in CCRT and sequential CRT

\begin{tabular}{lccc}
\hline $\begin{array}{c}\text { Tumor } \\
\text { characteristics }\end{array}$ & CCRT & $\begin{array}{c}\text { Sequential } \\
\text { CRT }\end{array}$ & p-value \\
\hline $\begin{array}{l}\text { Stage } \\
\text { I/II }\end{array}$ & 4 & 6 & 0.77 \\
III & 8 & 14 & \\
Node & & & 0.50 \\
0 & 3 & 18 & \\
$1-3$ & 9 & 8 & 0.95 \\
LVI & & 12 & \\
Negative & 5 & & 0.45 \\
Positive & 7 & 10 & \\
ENE & & 10 & \\
Negative & 8 & 4 & \\
Positive & 4 & & \\
\hline
\end{tabular}

$L V I$, Iymphovascular invasion; ENE, extranodal extension; CCRT, concurrent chemoradiation therapy; CRT, chemoradiotherapy.

Table 7. Incidence of treatment failure according to treatment scheme

\begin{tabular}{lcr}
\hline & \multicolumn{2}{c}{ No. of patients (\%) } \\
\cline { 2 - 3 } & LF & DM \\
\hline RT alone $(n=23)$ & $5(21.7)$ & $7(30.4)$ \\
CCRT $(n=12)$ & - & $6(50.0)$ \\
Sequential CRT $(n=20)$ & $3(15.0)$ & $10(50.0)$ \\
\hline
\end{tabular}

LF, locoregional failure; DM, distant metastasis; CCRT, concurrent chemoradiation therapy; CRT, chemoradiotherapy.

sequential chemoradiotherapy for treating NSCLC, CCRT demonstrates better treatment outcomes in local tumor control and OS. However, there are higher rates of acute grade 3 or greater esophagitis in patients who receive concurrent chemoradiotherapy in comparison with sequential combination chemoradiotherapy (relative risk [RR], 4.96; 95\% confidence interval [Cl], 2.17 to 11.37), and higher rates of grade 4 or greater neutropenia in the concurrent group (RR, $1.18 ; 95 \% \mathrm{Cl}_{1} 0.90$ to 1.55$)$ [10].

The results of our study show that CCRT can provide relatively better local tumor control than sequential CRT, but that sequential CRT also demonstrates good local tumor control. Distant metastasis did not decline following the administration of adjuvant chemotherapy in CCRT and sequential CRT groups, and sequential CRT can be safely applied to patients with poor performance, old age, or multiple morbidities (whereas CCRT cannot be applied in these situations). However, our study is limited by its retrospective design and the relatively small number of patients analyzed.

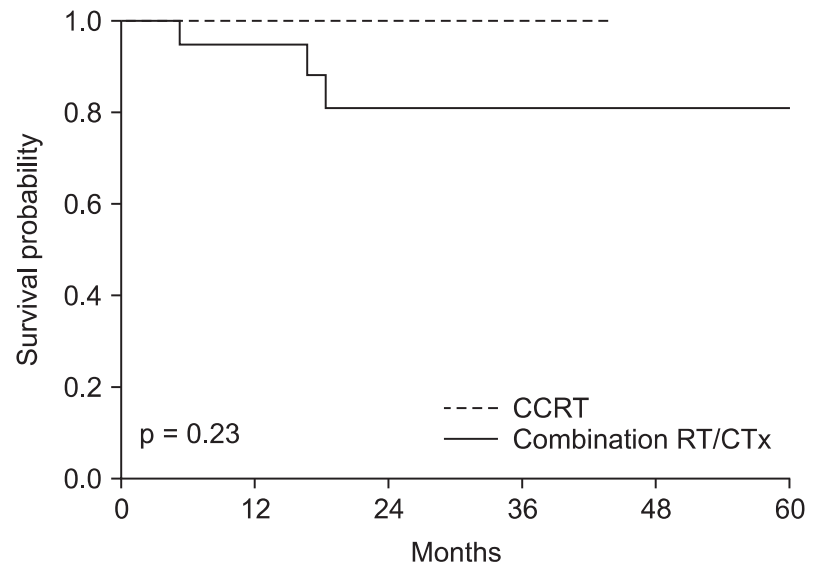

Fig. 3. Locoregional relapse-free survival rate in concurrent chemoradiation therapy (CCRT) vs. sequential chemoradiotherapy. $\mathrm{RT}$, radiation therapy; CTx, chemotherapy.

Administering PORT to incompletely resected NSCLC is an effective modality for improving local tumor control. Combination chemoradiotherapy can be better than PORT alone for local tumor control, but CCRT may be more effective than sequential CRT. Distant metastasis is still a major failure pattern in patients with incompletely resected NSCLC, even if combination chemoradiation is administered.

\section{Conflict of Interest}

No potential conflict of interest relevant to this article was reported.

\section{Acknowledgments}

This study was supported by a grant (2013-472) from the Asan Institute for Life Sciences, Seoul, Korea and by Basic Science Research Program through the National Research Foundation of Korea (NRF) funded by the Ministry of Education (No. 2013R1A1A2011346).

\section{References}

1. Kayser K, Anyanwu E, Bauer HG, Vogt-Moykopf I. Tumor presence at resection boundaries and lymph-node metastasis in bronchial carcinoma patients. Thorac Cardiovasc Surg 1993:41:308-11.

2. Ohguri T, Yahara K, Moon SD, et al. Postoperative radiotherapy for incompletely resected non-small cell lung cancer: clinical outcomes and prognostic value of the histological subtype. J Radiat Res 2012;53:319-25. 


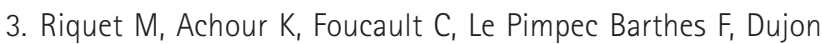
$A$, Cazes $A$. Microscopic residual disease after resection for lung cancer: a multifaceted but poor factor of prognosis. Ann Thorac Surg 2010;89:870-5.

4. Collaud S, Bongiovanni M, Pache JC, Fioretta G, Robert JH. Survival according to the site of bronchial microscopic residual disease after lung resection for non-small cell lung cancer. J Thorac Cardiovasc Surg 2009;137:622-6.

5. Karakoyun-Celik O, Yalman D, Bolukbasi Y, Cakan A, Cok G, Ozkok S. Postoperative radiotherapy in the management of resected non-small-cell lung carcinoma: 10 years' experience in a single institute. Int J Radiat Oncol Biol Phys 2010;76:4339.

6. Auperin A, Le Pechoux C, Rolland E, et al. Meta-analysis of concomitant versus sequential radiochemotherapy in locally advanced non-small-cell lung cancer. J Clin Oncol 2010;28:2181-90.
7. Wind J, Smit EJ, Senan S, Eerenberg JP. Residual disease at the bronchial stump after curative resection for lung cancer. Eur J Cardiothorac Surg 2007;32:29-34.

8. Ghiribelli C, Voltolini L, Paladini P, Luzzi L, Di Bisceglie M, Gotti $G$. Treatment and survival after lung resection for non-small cell lung cancer in patients with microscopic residual disease at the bronchial stump. Eur J Cardiothorac Surg 1999;16:5559.

9. Olszyna-Serementa M, Socha J, Wierzchowski M, Kepka L. Patterns of failure after postoperative radiotherapy for incompletely resected (R1) non-small cell lung cancer: implications for radiation target volume design. Lung Cancer 2013;80:179-84.

10. Feliciano J, Feigenberg S, Mehta M. Chemoradiation for definitive, preoperative, or postoperative therapy of locally advanced non-small cell lung cancer. Cancer J 2013;19:22230. 